\title{
Wind speed equalization-based incoming wind classification by aggregating DFIGs
}

\author{
Zhaojun MENG (ه), Feng XUE, Xueming LI
}

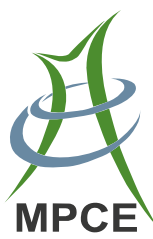

\begin{abstract}
With the development of wind energy, it is necessary to develop equivalent models to represent dynamic behaviors of wind farms in power systems. The equivalent wind method is investigated for the aggregation of doubly-fed induction generator wind turbines. The detailed procedures for the calculation of equivalent wind are analyzed. The necessity of classifying incoming winds is shown. To improve the performances of the method, incoming winds are classified according to mean wind speeds and positive/negative semi-variances of wind speeds, and a group of turbines with similar incoming winds are aggregated together. The effectiveness of the method is verified through simulations in MATLAB/Simulink.
\end{abstract}

Keywords DFIG, Power curve, Equivalent wind, Aggregated wind turbine

\section{Introduction}

With the increasing installed capacities for both individual wind turbines and wind farms, it is necessary to develop suitable models to represent dynamic behaviors of wind farms in power systems. For engineering applications, detailed models of all wind turbines in wind farms are computationally prohibitive. The equivalent wind farm models can reduce the model order and computation time, while the impacts of wind farms on power systems are appropriately represented for studies.

Received: 1 August 2012/Accepted: 8 October 2012/Published online: 5 July 2013

(C) The Author(s) 2013. This article is published with open access at Springerlink.com

Z. MENG, F. XUE, X. LI, NARI Group Corporation (State Grid Electric Power Research Institute), Nanjing 210003, Jiangsu, China

(凹) e-mail: xybeck@hotmail.com
Doubly-fed induction generator (DFIG) wind turbine has become the most widely used one in wind farms, since it has noticeable advantages, such as decoupled controls of active and reactive powers, and the use of a power converter with a rated power of $25 \%$ of total system power [1]. The equivalent methods for wind turbines have been studied in [1-5]. The method proposed in [2] is based on the dynamic simplification of each wind turbine, and the model of an equivalent wind turbine is different from that of individual wind turbine. The method proposed in $[1,3]$ divides the wind turbine model into two parts: a simplified model is used to represent the mechanical part of each wind turbine, and the generator parts of all wind turbines are aggregated into an equivalent generation system. The method requires non-negligible computation burden by solving those differential equations representing the mechanical parts of wind turbines.

The most commonly used equivalent method is to aggregate wind turbines with similar incoming winds; however, two problems arise in the implementation of the method. First, the criteria to classify incoming winds need to be defined. Second, incoming winds for equivalent wind turbines, need to be calculated. References $[4,5]$ proposed two methods: the first one is the average wind method, which sets a criterion, differences in wind speeds $<2 \mathrm{~m} / \mathrm{s}$, for the classification of incoming winds, and the average winds of the ones incident on aggregated wind turbines (AWTs) are used as equivalent winds. The second one is equivalent wind method (EWM), which aggregates all wind turbines into a single AWT, eliminating the need to classify any incoming winds. With EWM, the equivalent wind is derived from the power curves of AWT $[4,5]$. The performance of EWM is better than that of the average wind method since EWM can aggregate wind turbines with different incoming winds.

Based on built-in DFIG wind turbine models included in the SimPowerSystems library of MATLAB/Simulink 
$[6,7]$, EWM is investigated for the aggregation of DFIG wind turbines. The functions for defining the relationship between the turbine output power and the wind speed are derived, and a detailed procedure for the calculation of equivalent wind is provided. The necessity of classifying incoming winds is also shown. Besides, incoming winds are classified according to the mean wind speeds (MWSs) and the positive/negative semi-variances of wind speeds (VWSs). A group of turbines with similar wind are aggregated together. The effectiveness of the method is demonstrated through simulations.

\section{Power curves of DFIG wind turbine}

DFIG wind turbine uses a wound rotor induction generator with the stator connected directly to the grid and the rotor connected to the grid via two back-to-back IGBT converters. The dynamic behavior of a DFIG wind turbine is represented by the following models: rotor, drive train, induction generator, converter, and protection in systems, rotor speed controller represented by the power curve, and blade pitch angle controller in $[1,4]$. A detailed description of these models can be found in [6-9]. This paper only concentrates on the power curves of DFIG wind turbine.

Fig. 1 shows the power curves of the DFIG wind turbine model from SimPowerSystems [6, 7]. Each point for the power curves has three values: turbine output power, wind speed, and turbine speed. When the incoming wind is less than the nominal wind speed (NWS), DFIG uses the optimal tracking strategy (OPTS) to capture the maximum wind energy, which corresponds to the curve between point $B$ and point $C$. When the incoming wind is greater than NWS, the blade pitch control is used to reduce the mechanical power to the equipment rating, which corresponds to the horizontal line starting from point $D$. The curve between point $C$ and point $D$ is to smooth the power fluctuations occurring near the NWS [10]. When the incoming wind speed is lower than that of point $A$, the mechanical power of the DFIG is zero, and the curve between point $A$ and point $B$ is to smooth the power fluctuations occurring near point $A$.

Table 1 shows the statistics regarding to the four points $A, B, C$, and $D$ of the power curves.

The derivation of the turbine output power versus wind speed (PVW) function is shown as follows: at point $C$, the wind speed is $v_{\mathrm{NWS}}$, turbine speed is nominal turbine speed $\omega_{\text {nom }}$ (in p.u. of generator synchronous speed), and the turbine output power in p.u. is $k_{\mathrm{p}}$. For a given wind speed $v_{\text {wind_pu }}=v_{\text {wind }} / v_{\mathrm{NwS}}$, the turbine output power in p.u. is given by [7]:

$P_{\mathrm{m} \_\mathrm{pu}}=k_{\mathrm{p}} c_{\mathrm{p} \_\mathrm{pu}} v_{\text {wind_pu }}^{3}$,

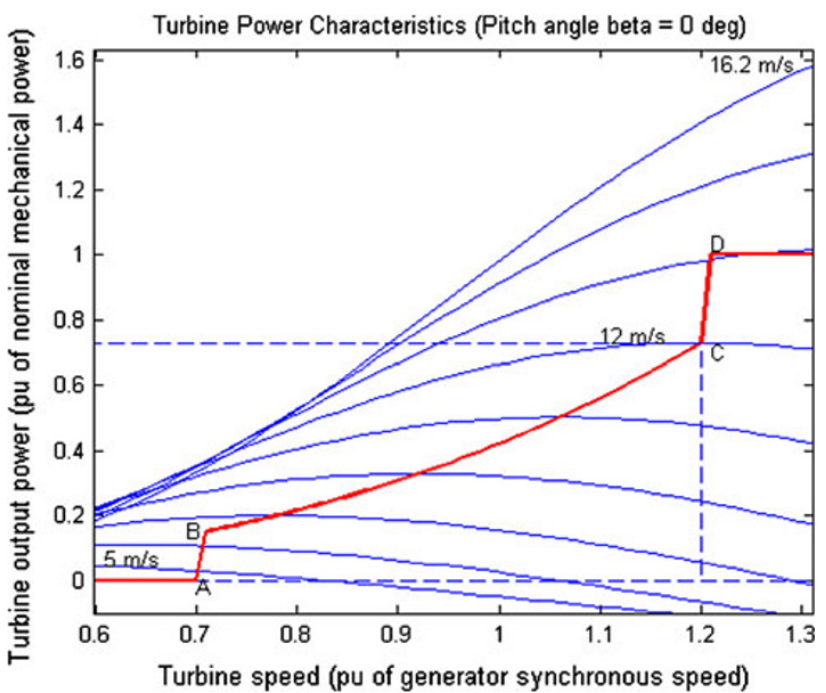

Fig. 1 Power curves of DFIG from SimPowerSystems

Table 1 Statistics of points $A, B, C$, and $D$

\begin{tabular}{llll}
\hline Point & $\begin{array}{l}\text { Wind } \\
\text { speed/(m/s) }\end{array}$ & $\begin{array}{l}\text { Turbine } \\
\text { speed/(p.u.) }\end{array}$ & $\begin{array}{l}\text { Turbine output } \\
\text { power/(p.u.) }\end{array}$ \\
\hline$A$ & 4.23 & 0.7 & 0 \\
$B$ & 7.1 & 0.71 & 0.151 \\
$C$ & 12 & 1.2 & 0.73 \\
$D$ & 13.48 & 1.21 & 1.0 \\
\hline
\end{tabular}

where $c_{\mathrm{p} \_\mathrm{pu}}=c_{\mathrm{p}} / c_{\mathrm{p} \_ \text {max }}$ is the power coefficient in p.u.; $c_{\mathrm{p} \_ \text {max }}=0.48$ is the maximum power coefficient; $c_{\mathrm{p}}$ is defined by

$c_{\mathrm{p}}=c_{1} \cdot\left(\frac{c_{2}}{\lambda^{\prime}}-c_{3} \cdot \beta-c_{4}\right) \cdot e^{\frac{-c_{5}}{\lambda^{\prime}}}+c_{6} \cdot \lambda$,

where $\lambda$ is the tip ratio speed, given by

$\lambda=\frac{\omega_{\mathrm{r} \_ \text {pu }} / \omega_{\text {nom }}}{v_{\text {wind } \_ \text {pu }}} \cdot \lambda_{\text {nom }}=K_{\text {nom }} \frac{\omega_{\text {r_pu }}}{v_{\text {wind_pu }}}$,

$\frac{1}{\lambda^{\prime}}=\frac{1}{\lambda+0.08 \cdot \beta}-\frac{0.035}{\beta^{3}+1}=\frac{1}{\lambda}-0.035$,

where $\beta=0$ is the nominal pitch angle; and $\omega_{\mathrm{r} \_p u}$ is the turbine speed in p.u. of generator synchronous speed.

In this paper, the following parameters have been used for the calculation of $c_{\mathrm{p}}$ [7]:

$c_{1}=0.5176, c_{2}=116, c_{3}=0.4, c_{4}=5, c_{5}=21$, $c_{6}=0.0068$, and $\lambda_{\text {nom }}=8.1$.

Substituting (2) and (4) into (1), the equation can be derived as

$P_{\mathrm{m} \_\mathrm{pu}}=f\left(\omega_{\mathrm{r} \_\mathrm{pu}}, v_{\text {wind } \_ \text {pu }}\right)$.

Since $\omega_{\mathrm{r}}$ pu is defined for each part of the power curve, (5) can be derived as the PVW function. 
2.1 PVW function for the power curve between point $B$ and point $C$

According to the OPTS, $\omega_{\mathrm{r} \_ \text {pu }}$ can be calculated through the solution of $\partial C_{\mathrm{p}} / \partial \omega_{\mathrm{r} \_\mathrm{pu}}=0$. However, this calculation is unnecessary since power coefficients $c_{\mathrm{p}}$ is $c_{\mathrm{p} \_ \text {max }}$ for the OPTS. Therefore, the PVW function for the power curve between point $B$ and point $C$ is given by

$P_{\mathrm{m} \_\mathrm{pu}}=k_{p} v_{\text {wind_pu }}^{3}$.

2.2 PVW function for the power curve between point $C$ and point $D$

In this case, $\omega_{\mathrm{r} \_ \text {pu }}$ is given by

$\omega_{\mathrm{r} \_\mathrm{pu}}=\frac{\omega_{D}-\omega_{C}}{P_{D}-P_{C}} \cdot\left(P_{\mathrm{m} \_\mathrm{pu}}-P_{C}\right)+\omega_{C}$,

where $P_{C}$ and $P_{D}$ are turbine output powers (p.u.) at point $C$ and point $D$, and $\omega_{C}$ and $\omega_{D}$ are turbine speeds (p.u.) at point $C$ and point $D$, respectively.

Substituting (7) into (5), the PVW function for the power curve between point $C$ and point $D$ can be derived as

$P_{\mathrm{m} \_\mathrm{pu}}=g\left(P_{\mathrm{m} \_\mathrm{pu}}, v_{\text {wind } \_ \text {pu }}\right)$.

2.3 PVW Function for the power curve between point $A$ and point $B$

In this case, $\omega_{\mathrm{r} \_\mathrm{pu}}$ is given by

$\omega_{\mathrm{r} \_\mathrm{pu}}=\frac{\omega_{B}-\omega_{A}}{P_{B}-P_{A}} \cdot\left(P_{\mathrm{m} \_\mathrm{pu}}-P_{A}\right)+\omega_{A}$,

where $P_{A}$ and $P_{B}$ are turbine output powers (p.u.) at point $A$ and point $B$, and $\omega_{C}$ and $\omega_{D}$ are turbine speeds (p.u.) at point $C$ and point $D$, respectively.

Substituting (9) into (5), the PVW function for the power curve between point $A$ and point $B$ can be derived as

$P_{\mathrm{m} \_\mathrm{pu}}=h\left(P_{\mathrm{m} \_\mathrm{pu}}, v_{\text {wind } \_\mathrm{pu}}\right)$.

\section{Equivalent wind method}

EWM proposed in $[4,5]$ is used for the aggregation of DFIG wind turbines, and its procedures are listed as follows:

(1) Based on its PVW function, the power $P_{j}^{\mathrm{wt}}$ generated by each wind turbine is calculated according to its incoming wind.

(2) The power generated by the equivalent wind turbine is equal to the sum of $P_{j}^{\mathrm{wt}}$,

$P_{\mathrm{eq}}^{\mathrm{wt}}=\sum_{j=1}^{n} P_{j}^{\mathrm{wt}}$.
After that, $P_{\text {eq }}^{\mathrm{wt}}$ is expressed in the equivalent wind turbine base as $P_{\mathrm{eq}}^{\text {ewt }}$.

(3) Based on the PVW function of the equivalent wind turbine, which are the same as that of a single wind turbine, the equivalent wind $v_{\text {eq }}$ is calculated. Depending on the value of $P_{\text {eq }}^{\text {ewt }}$, solutions of (5), (8) or (10) are involved. Newton method can be used for this purpose. For online applications, a look up table can be calculated offline to save computational efforts.

The original work of EWM [4, 5], which is referred to as EWM1, aggregates all the wind turbines into a single AWT. However, different parts of the power curve have completely different characteristics, which can lead to poor performance for EWM1. To overcome this shortcoming, an improved method is proposed in [11], turbines with MWSs belonging to the same part of the power curve are aggregated together. However, if two turbine MWSs are close to each other, but the VWSs differ significantly, then the aggregation will cause great errors. Therefore, the effects of VWS cannot be neglected. To achieve good performance for aggregation, wind turbines experiencing similar incoming wind should be aggregated together. Two statistical values of wind speed, MWS and VWS are used to quantify this similarity. The conventional calculation of variances [12] only measures the absolute values of the differences between instantaneous values and the mean values (DBIMs). To improve the performance of aggregation, two factors need to be considered in the calculation of variances: (1) it is necessary to distinguish between positive and negative values of DBIM; (2) wind speed fluctuates continuously, which incurs continuous changes under operational conditions of wind turbines, so DBIMs at different time must be distinguished from each other. Positive semi-variance $\sigma_{+}$and negative semi-variance $\sigma_{-}$are introduced as follows:

$\sigma_{+}=\sqrt{\frac{\sum_{t \in \sigma_{+}} w_{t}\left(v_{t}-\bar{v}\right)^{2}}{\sum_{t=t_{0}}^{t_{\mathrm{n}}} w_{t}}}$,

where $t \in \sigma_{+}$includes all the time $t$, which satisfies the condition of $v_{t}>\bar{v} ; \bar{v}$ is the MWS between $t_{0}$ and $t_{\mathrm{n}}$. A weight $W_{t}$ varying with the time $t$ is given by

$w_{t}=\frac{1.0}{1.0+\frac{t}{K}}$,

where $t / K>0$ is used to distinguish among DBIMs at different time, and $K=0.5$ is used for demonstration. For use of the proposed method, a deep investigation is needed for a more efficient formulation of $W_{t}$.

$\sigma_{-}$is given by

$\sigma_{-}=\sqrt{\frac{\sum_{t \in \sigma_{-}} w_{t}\left(v_{t}-\bar{v}\right)^{2}}{\sum_{t=t_{0}}^{t_{\mathrm{n}}} w_{t}}}$, 
where $t \in \sigma_{-}$includes all the time $t$, which satisfies the condition of $v_{t}<\bar{v}$; $\bar{v}$ is the MWS between $t_{0}$ and $t_{\mathrm{n}}$.

Based on the calculated MWSs, $\sigma_{+}$and $\sigma_{-}$, two grouping strategies are used for the aggregation: one is more relaxing (referred to as RS) and the other is stricter (referred to as SS).

(1) For wind turbines with $\sigma_{+}<\sigma_{\mathrm{th} p 1}$ and $\sigma_{-}<\sigma_{\mathrm{th} n 1}$ ( $\sigma_{\mathrm{th} p 1}$ and $\sigma_{\mathrm{th} n 1}$ are thresholds to distinguish between RS and SS), RS is used for the aggregation. The RS aggregates wind turbines experiencing MWSs belonging to the same parts of the power curve. Since large power fluctuations occur near NWS, the RS aggregates wind turbines into three AWTs: the first AWT represents wind turbines with MWS less than $v_{C}$, the second AWT represents wind turbines with MWSs between $v_{C}$ and $v_{D}$, and the third AWT represents wind turbines with MWSs above $v_{D}$, where $v_{C}$ and $v_{D}$ are wind speeds at points $C$ and $D$ of Fig. 1 .

The equivalent wind for the third AWT is calculated as the average wind, since the power curve is limited to the rated power, any wind above $v_{D}$ is the solution $[4,5]$. The RS neglects power fluctuations occurring near $v_{A}$ due to their small magnitudes. If one of the three AWTs does not have any associated wind turbines, the AWT will be omitted in the implementation.

(2) For wind turbines with $\sigma_{+} \geq \sigma_{\mathrm{th} p 1}$ or $\sigma_{-} \geq \sigma_{\mathrm{th} n 1}$, SS is used for the aggregation. Any two turbines within a group, which can be aggregated together, must meet the following three criteria, we have

$\left|\overline{v_{i}}-\overline{v_{j}}\right|<v_{\mathrm{th}}$,

where $\overline{v_{i}}$ is the $i$ th turbine's MWS; $\overline{v_{j}}$ is the ith turbine MWS; and $v_{\text {th }}$ is a threshold;

$\left|\sigma_{i+}-\sigma_{j+}\right|<\sigma_{\text {thp } 2}$,

where $\sigma_{i+}$ is the $i$ th turbine $\sigma_{+} ; \sigma_{j+}$ the $j$ th turbine $\sigma_{+}$; and $\sigma_{\text {thp } 2}$ is a threshold.

$\left|\sigma_{i-}-\sigma_{j-}\right|<\sigma_{\mathrm{th} n 2}$, where $\sigma_{i-}$ is the $i$ th turbine $\sigma_{-} ; \sigma_{j-}$ is the $j$ th turbine $\sigma_{-}$; and $\sigma_{\mathrm{th} n 2}$ is a threshold.

EWM with the proposed grouping strategies is referred to as EWM2 for convenience. The following parameters of the EWM2 are used in this paper for demonstration: $\sigma_{\mathrm{th} p 1}=1.0, \sigma_{\mathrm{th} n 1}=1.0, v_{\mathrm{th}}=0.2(\mathrm{~m} / \mathrm{s}), \sigma_{\mathrm{th} p 2}=0.2$, and $\sigma_{\mathrm{th} n 2}=0.2$. For applications of EWM2, further investigation of these threshold settings is needed.

\section{Simulation results}

A $9 \mathrm{MW}$ wind farm from SimPowerSystems has been used for studies. As shown in Fig. 2, the wind farm consists of six 1.5 MW wind turbines connected to a $25 \mathrm{kV}$ distributed system, and a $500 \mathrm{~kW}$ load is connected to the $575 \mathrm{~V}$ bus of the wind farm. The parameters of the system can be found in [6]. To evaluate the EWM1 and EWM2 representing the collective responses of the wind farm in power systems, the dynamic responses of equivalent models are compared during a normal operation and a grid disturbance.

\subsection{Normal operations}

During normal operations, all the wind turbines in the wind farm operate on wind fluctuations. Two cases are presented. Table 2 summarizes the statistics of wind speed incidents on wind turbines.

Figure 3 shows the incoming wind for all wind turbines. At $T=5 \mathrm{~s}$, wind ramp occurs for WT6, which causes large value of $\sigma_{-}$. Therefore, WT6 needs to be handled by $\mathrm{SS}$, and the rest of wind turbines are handled by RS. As shown in Figs. 4 and 5, the dynamic responses of EWM2 are consistent well with those of the detailed models. Besides, the performance of EWM1 is much worse than that of EWM2.

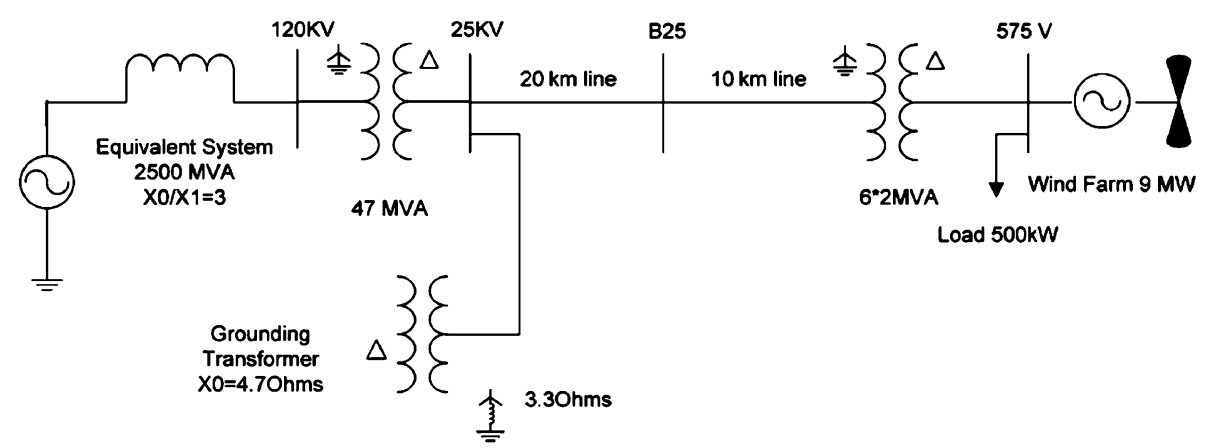

Fig. 29 MW wind farm connected to a distributed system 
Table 2 Statistics of wind speed incidents on wind turbine (WT)

\begin{tabular}{lllllll}
\hline WT & WT1 & WT2 & WT3 & WT4 & WT5 & WT6 \\
\hline MWS & 13.94 & 14.78 & 11.99 & 10.03 & 9.07 & 12.9 \\
$\sigma_{+}$ & 0.20 & 0.18 & 0.16 & 0.39 & 0.67 & 0.56 \\
$\sigma_{-}$ & 0.15 & 0.50 & 0.19 & 0.14 & 0.18 & 3.60 \\
\hline
\end{tabular}

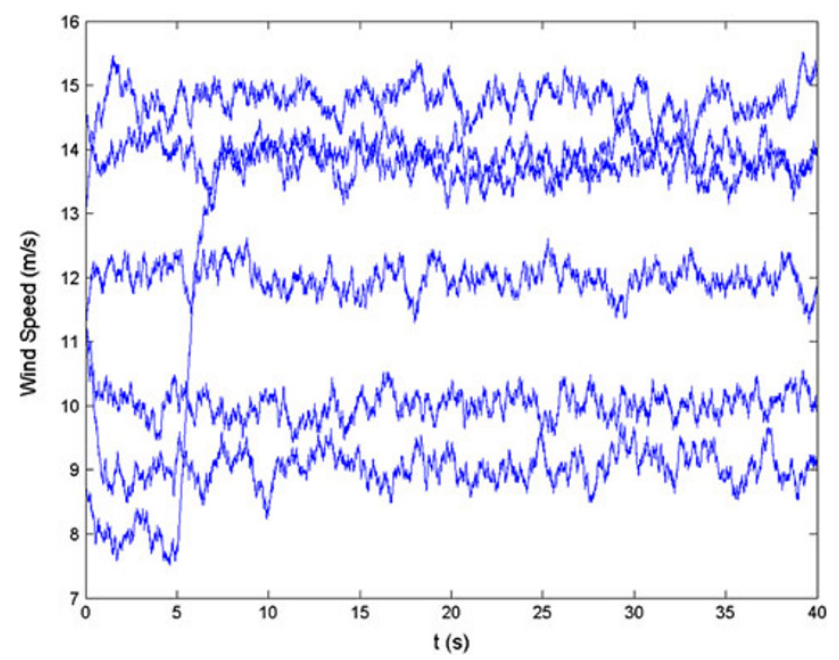

Fig. 3 Wind fluctuations

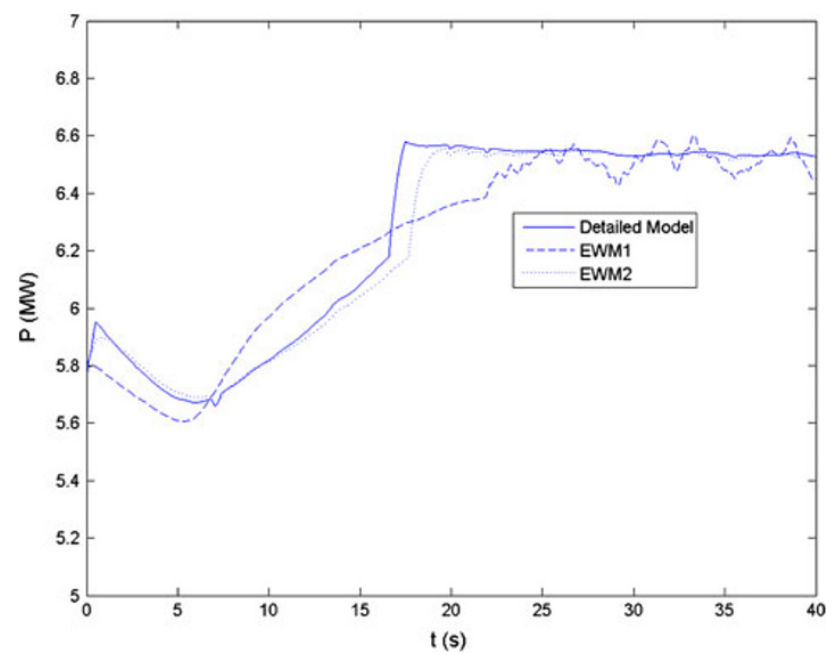

Fig. 4 Generated active power of the wind farm (normal states)

\subsection{Grid disturbance}

In this case, when $t=2 \mathrm{~s}$, a three phase short circuit of 8 cycle $(0.133 \mathrm{~s})$ is applied at bus B25. Because the grid disturbances are much faster than wind speed variations,

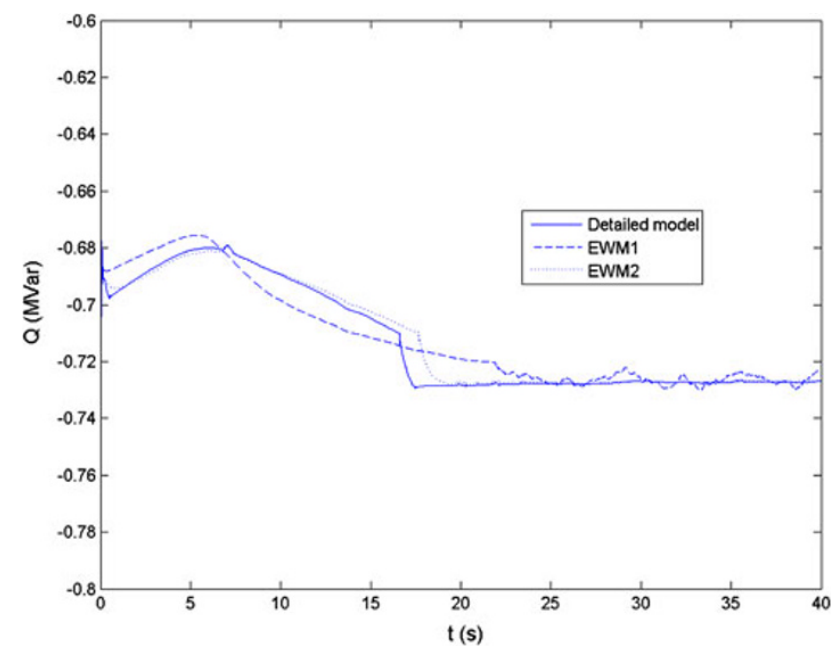

Fig. 5 Generated reactive power of the wind farm (normal states)

Table 3 Statistics of winds

\begin{tabular}{llcllll}
\hline WT & WT1 & WT2 & WT3 & WT4 & WT5 & WT6 \\
\hline Wind speed (m/s) & 6 & 6 & 10 & 10 & 14 & 14 \\
EWM1 & $v_{\text {eq }}=10.59$ & & & & \\
EWM2 & $v_{\text {eq1 }}=8.43$ & & & & $v_{\text {eq2 }}=14$ \\
\hline
\end{tabular}

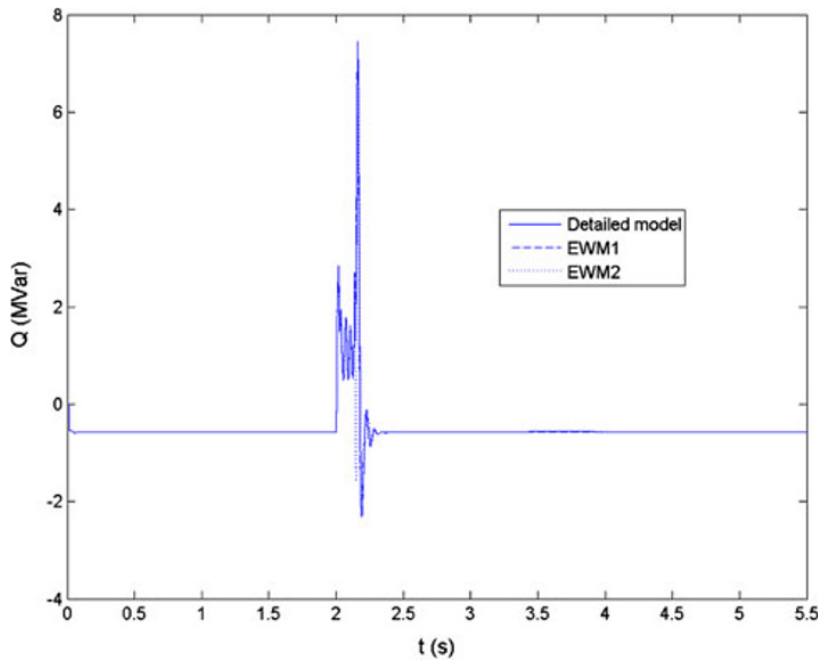

Fig. 6 Generated reactive power of the wind farm (with fault)

wind incidents on wind turbines are constantly assumed [4]. Table 3 summarizes the equivalent winds calculated by EWM1 and EWM2.

Although an accurate correspondence among the detailed model and both EWMs for the generated reactive power can be observed in Fig. 6. Fig. 7 shows that the EWM1 fails to capture active power fluctuations occurring in the detailed model when $t=3.4 \mathrm{~s}$, while EWM2 successfully captures 


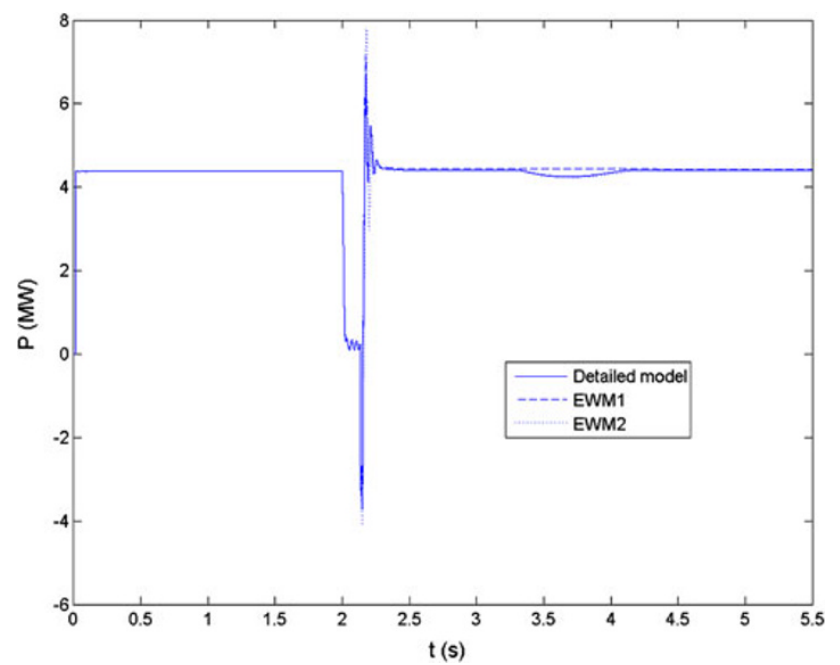

Fig. 7 Generated active power of the wind farm (with fault)

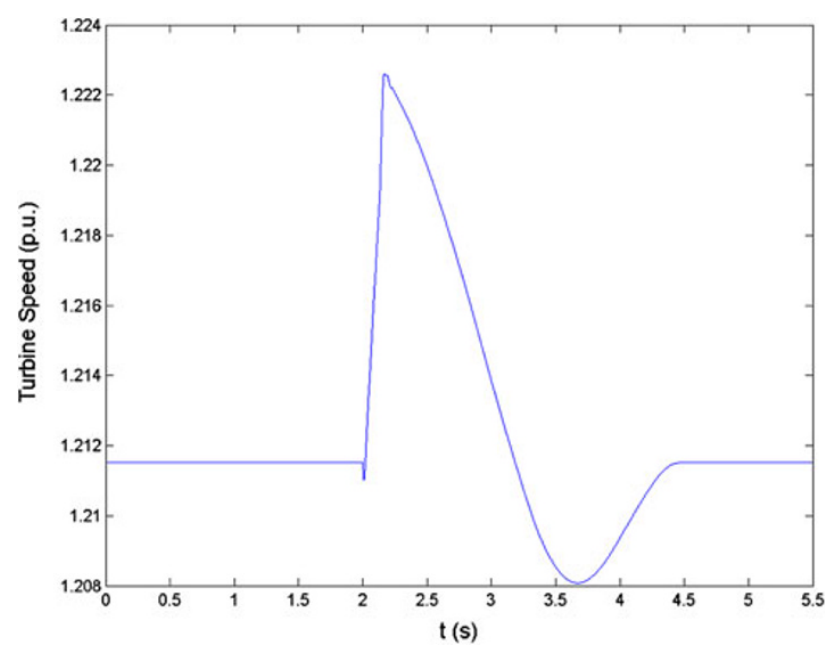

Fig. 8 Turbine speed of WT5 (WT6) in p.u.

this phenomenon. As shown in Fig. 8, during active power fluctuations, the turbine speeds of WT5 and WT6 are less than $v_{D}=1.21$, which means that the mechanical outputs of WT5 and WT6 are less than the rated power. When the turbine speeds of WT5 and WT6 are recovered above $v_{D}=1.21$, the mechanical outputs of WT5 and WT6 are set back to the rated power to end the active power fluctuations.

\section{Conclusion}

The equivalent wind method has been investigated for the aggregation of DFIG wind turbines. The detailed procedures for the calculation of equivalent wind are analyzed. The necessity of classifying incoming winds is also illustrated. In order to improve the performance of the equivalent wind method, incoming winds are classified according to the
MWSs and the positive/negative semi-VWSs. A group of turbines with similar wind are aggregated. The effectiveness of the proposed strategy is verified through simulations in MATLAB/Simulink. The aggregation technique is applicable for the studies of the impacts of wind farm integration on power system stability. Future work is needed to investigate its applications in large scale power systems, thus the proposed method needs to be studied, which includes a more efficient formula for the calculation of semi-variances and threshold settings for incoming wind classification.

Acknowledgments This study was supported by Science Foundation of Jiangsu Province (No. BK2011137), National Key Technology R\&D Program (No. 2011BAA07B03), State Grid Corporation of China, 2012 research and demonstration project on the key technologies for large scale grid friendly wind farms.

Open Access This article is distributed under the terms of the Creative Commons Attribution License which permits any use, distribution, and reproduction in any medium, provided the original author(s) and the source are credited.

\section{References}

[1] Fernandez LM, Saenz JR, Jurado F (2008) Aggregated dynamic model for wind farms with doubly fed induction generator wind turbines. Renew Energy 33(1):129-140

[2] Slootweg JG, Kling WL (2003) Aggregated modelling of wind parks in power system dynamics simulations. In: 2003 IEEE Bologna power tech conference proceedings, vol 3, Bologna, Italy, 25-29 Jul 2003, p 6

[3] Fernandez LM, Saenz JR, Jurado F (2006) Dynamic models of wind farms with fixed speed wind turbines. Renew Energy 31(8):1203-1230

[4] Fernandez LM, Garcia CA, Saenz JR et al (2006) Reduced model of DFIGs wind farms using aggregation of wind turbines and equivalent wind. In: Proceedings of the 2006 IEEE Mediterranean electrotechnical conference (MELCON'06), Benalmádena, Spain, 16-19 May 2006, pp 881-884

[5] Fernandez LM, Garcia CA, Saenz JR et al (2009) Equivalent models of wind farms by using aggregated wind turbines and equivalent winds. Energy Conserv Manag 50(3):691-704

[6] SimPowerSystems user's guide (2009) The Mathworks Inc, Natick

[7] SimPowerSystems reference (2009) The Mathworks Inc, Natick

[8] Pena R, Clare JC, Asher GM (1996) Doubly fed induction generator using back-to-back PWM converters and its applications to variable-speed wind-energy generation. IEE Proc Electr Power Appl 143(3):231-241

[9] Slootweg JG, Polinder H, Kling WL (2003) Representing wind turbine electrical generating systems in fundamental frequency simulations. IEEE Trans Energy Convers 18(4):516-524

[10] Slootweg JG, de Haan SWH, Polinder H et al (2003) General model for the representing variable speed wind turbines in power system dynamic simulations. IEEE Trans Power Syst 18(1):144-151

[11] Meng ZJ, Xue F (2010) An investigation of the equivalent wind method for the aggregation of DFIG wind turbine. In: Proceedings of the 2010 Asia-Pacific power and energy engineering, Chengdu, China, 28-31 Mar 2010, p 6

[12] Statistics toolbox user's guide (2009) The Mathworks Inc, Natick 


\section{Author Biographies}

Zhaojun MENG obtained B.Eng. from North China Institute of Electric Power, M.Eng. from Wuhan University of Hydraulic and Electric Engineering and Nanyang Technological University, and $\mathrm{Ph} . \mathrm{D}$. from the University of Strathclyde. His main research field is power system analysis and control.
Feng XUE obtained his Ph.D. degree from the University of Bath, UK in 2008. His main research field is power system analysis and control.

Xueming LI obtained his M.Sc. from State Grid Electric Power Research Institute in 1989. His main research field is power system stability analysis and control. 